\title{
Gemcitabine plus metronomic 5-fluorouracil or capecitabine as a second-/third-line chemotherapy in advanced adrenocortical carcinoma: a multicenter phase II study
}

\begin{abstract}
Paola Sperone ${ }^{1}$,Anna Ferrero ${ }^{1}$, Fulvia Daffara ${ }^{2}$, Adriano Priola ${ }^{3}$, Barbara Zaggia ${ }^{2}$, Marco Volante ${ }^{4}$, Daniele Santini ${ }^{5}$, Bruno Vincenzi ${ }^{5}$, Giuseppe Badalamenti ${ }^{6}$, Chiara Intrivici ${ }^{6}$, Sabrina Del Buono ${ }^{7}$, Silvia' De Francia ${ }^{8}$, Emmanouil Kalomirakis ${ }^{9}$, Riccardo Ratti ${ }^{10}$, Alberto Angeli ${ }^{2}$, Luigi Dogliotti ${ }^{1}$, Mauro Papotti ${ }^{4}$, Massimo Terzolo ${ }^{2}$ and Alfredo Berruti ${ }^{1}$
\end{abstract}

\footnotetext{
${ }^{1}$ Oncologia Medica, ${ }^{2}$ Medicina Interna, ${ }^{3}$ Radiologia and ${ }^{4}$ Anatomia Patologica, Dipartimento di Scienze Cliniche e Biologiche Università di Torino, Orbassano, Italy

${ }^{5}$ Oncologia Medica, Università Campus Biomedico, Roma, Italy

${ }^{6}$ Oncologia Medica, Policlinico Giaccone, Palermo, Italy

${ }^{7}$ Oncologia Medica, Ospedale di Arezzo, Arezzo, Italy

${ }^{8}$ Farmacologia, Dipartimento di Scienze Cliniche e Biologiche, Università di Torino, Orbassano, Italy

${ }^{9}$ Endocrinologia, Azienda Ospedaliera Cardarelli, Napoli, Italy

${ }^{10}$ Oncologia Medica, Ospedale di Sanremo, Sanremo, Italy
}

(Correspondence should be addressed to P Sperone who is now at Oncologia Medica, Azienda Ospedaliero-Universitaria San Luigi Gonzaga, Regione Gonzole 10, 10043 Orbassano, Italy; Email: paola.sperone@email.it)

\begin{abstract}
Adrenocortical carcinoma (ACC) is a rare neoplasm characterized by poor prognosis. First-line systemic treatments in advanced disease include mitotane, either alone or in combination with chemotherapy. Studies evaluating second-line therapy options have obtained disappointing results. This trial assessed the activity and toxicity of gemcitabine plus metronomic fluoropyrimidines in heavily pretreated advanced ACC patients. From 1998 to 2008, 28 patients with advanced ACC progressing after mitotane plus one or two systemic chemotherapy lines were enrolled. They received a combination of i.v. gemcitabine $\left(800 \mathrm{mg} / \mathrm{m}^{2}\right.$, on days 1 and 8 , every 21 days $)$ and i.v. 5 -fluorouracil protracted infusion $\left(200 \mathrm{mg} / \mathrm{m}^{2} /\right.$ daily without interruption until progression) in the first six patients, or oral capecitabine $(1500 \mathrm{mg} /$ daily $)$ in the subsequent patients. Mitotane administration was maintained in all cases. The rate of non-progressing patients after 4 months of treatment was $46.3 \%$. A complete response was observed in 1 patient $(3.5 \%)$; 1 patient $(3.5 \%)$ obtained a partial regression, 11 patients (39.3\%) obtained a disease stabilization and 15 patients (53.7\%) progressed. Treatment was well tolerated, with grade III and IV toxicities consisting of leukopenia in six patients (21.4\%), thrombocytopenia in one patient (3.5\%), and mucositis in one patient (3.5\%). Median time to progression and overall survival in the patient population were 5.3 (range: 1-43) and 9.8 months (range: $3-73$ ) respectively. Gemcitabine plus metronomic fluoropyrimidines is a well-tolerated and moderately active regimen in heavily pretreated ACC patients.
\end{abstract}

Endocrine-Related Cancer (2010) 17 445-453

\section{Introduction}

Adrenocortical carcinoma (ACC) is an extremely rare disease characterized by poor prognosis (Crucitti et al. 1996, Icard et al. 2001). The overall 5-year survival rate of ACC patients ranges between 23 and $60 \%$ (Icard et al. 2001, Abiven et al. 2006). Stage at diagnosis is the strongest prognostic parameter (Allolio \& Fassnacht 2006, Fassnacht et al. 2009), and surgery 
is the mainstay of treatment. However, a significant number of patients are not operable at diagnosis, and many patients radically resected are destined to relapse frequently within the first 2 years (Allolio \& Fassnacht 2006). The prognosis of advanced/metastatic ACC patients not amenable to surgery is dismal with an estimated survival rate ranging within few months and few years (Crucitti et al. 1996, Icard et al. 2001). Systemic therapy for ACC at this stage has lead to modest results, and it is actually not clear whether it has any role in modifying the natural history of the disease. The adrenolytic drug mitotane (1,1 dichloro- 2 (o-chlorophenyl)-2-( $p$-chloro-phenyl) ethane) has been widely employed, but objective responses were observed in about $25 \%$ of cases only (Hahner \& Fassnacht 2005). Advanced ACC patients progressing to mitotane treatment or patients demonstrating a rapid pattern of tumor growth are usually treated with chemotherapy. However, experience with cytotoxic chemotherapy is limited. Only nine phase II studies of chemotherapy including ten or more patients have been published up to now (van Slooten \& van Oosterom 1983, Decker et al. 1991, Schlumberger et al. 1991, Bukowski et al. 1993, Bonacci et al. 1998, Khan et al. 2000, Williamson et al. 2000, Abraham et al. 2002, Berruti et al. 2005). Cisplatin is the cytotoxic agent most frequently used either alone or in combination with mitotane or other cytotoxic drugs. Cisplatincontaining regimens lead to response rates ranging between 11 and $48 \%$. Combination chemotherapeutic regimens including mitotane seem to be more active than those without. Whatever was the cytotoxic regimen employed, the median duration of disease response ranged between 7 and 18 months. The question of whether cisplatin-containing schemes are more efficacious than non-cisplatin-containing ones is being addressed by a large multicenter, multinational, randomized prospective study that is currently ongoing with the aim to compare efficacy of etoposide, doxorubicin, and cisplatin (EDP) plus mitotane versus streptozotocin plus mitotane (FIRM-ACT study). On these premises, many patients submitted to chemotherapy and mitotane are expected to fail or progress after initial response, and there is, therefore, an urgent need for salvage therapy. At the present time, a few experiences regarding the role of second- and thirdline therapy for refractory ACC have shown disappointing results (Baudin et al. 2002, Khan et al. 2004, Quinkler et al. 2008, Wortmann et al. 2009). At the Consensus Conference held in Ann Arbor in 2003 (Schteingart et al. 2005), gemcitabine was considered among the most promising agents. The combination of gemcitabine and fluoropyrimidines, such as 5-fluorouracil and capecitabine, has been found to be synergistic and efficacious in the management of advanced pancreatic cancer (Herrmann et al. 2007). The combination of gemcitabine and capecitabine has shown a modest but reproducible activity in patients with advanced kidney cancer, a disease notoriously resistant to chemotherapy (Tannir et al. 2008).

Metronomic chemotherapy is the frequent administration of cytotoxic drugs at doses that are low enough to avoid dose-limiting adverse effects, which would otherwise require rest periods (Kerbel \& Kamen 2004). This treatment modality targets tumor cells indirectly via inhibiting angiogenesis and vasculogenesis by continuously exposing the more slowly proliferating tumor endothelial cells to cytotoxic therapy (Bottini et al. 2006). Low-dose metronomic chemotherapy may offer several advantages, including low toxicity and treatment response irrespective of the resistance profile of the tumor cell population (Berruti et al. 2000). Based on these observations, we have designed and conducted a phase II study with the aim of testing the activity of gemcitabine administered in association with continuous 5-fluorouracil infusion or metronomic capecitabine, an oral fluoropyrimidine derivative, as a second-/third-line approach in advanced ACC patients.

\section{Patients and methods}

Patients with histologically confirmed diagnosis of ACC, locally advanced or metastatic not amenable to radical resection were considered eligible. Central pathology review was not mandatory before patient registration in the study, but in all cases, the histological diagnosis of ACC was confirmed according to the Weiss criteria (Weiss 1984) by the study pathologist. Inclusion criteria were at least one radiologically measurable lesion, progressive disease after one or two cytotoxic chemotherapy lines (including a cisplatin-based protocol), performance status $0-2$ according to ECOG scale, life expectancy $\geq 3$ months, age $\geq 18$ years, adequate bone marrow reserve (neutrophils $\geq 1500 / \mathrm{mm}^{3}$ and platelets $\geq 80000 / \mathrm{mm}^{3}$ ), adequate renal and hepatic function (creatinine clearance $>60 \mathrm{ml} / \mathrm{min}$, bilirubin, aspartate aminotransferase, and alanine aminotransferase $\leq 1.5$ times the upper limit of normality), and effective contraception in pre-menopausal females. Exclusion criteria were previous exposure to gemcitabine or fluoropyrimidines, history of other neoplasms within 5 years, presence of active infection, history of myocardial infarction or unstable angina, cardiac insufficiency, uncontrolled arrhythmias, presence of osteoblastic bone metastasis as the only site of disease, 
and radiation therapy to measurable lesions. The study was approved by the local ethical committees of the participating centers. Written informed consent was obtained from all patients before inclusion in the study.

\section{Patient evaluations}

Pre-chemotherapy evaluations included a documentation of complete medical oncologic history, physical examination, assessment of performance status (ECOG scale), routine chemistry, determination of steroid hormone levels (including at least measurement of serum and urinary cortisol, dehydroepiandrosterone sulfate and, in women, testosterone), electrocardiography, computed tomography (CT) scanning of chest and abdomen, and bone scan if the presence of bone metastases was clinically suspected. Physical examination and routine chemistry were repeated at days 1 and 8 of every cycle.

\section{Evaluation of response and toxicity}

Assessment of response was initially planned to be performed according to the WHO criteria (Miller et al. 1981). From 2000 onwards, however, RECIST criteria were introduced (Therasse et al. 2000), and patients previously evaluated with WHO criteria were retrospectively reassessed according to RECIST. Hormone response was defined as complete if a normalization of all previously elevated steroids was attained, while partial hormone response was defined as a reduction $>50 \%$ of pretreatment levels. However, changes in hormone levels per se were not considered as a response to treatment. Tumor status and endocrine pattern were assessed after 2 and 4 months for the first two evaluations and then every 3 months, until discontinuation of study treatment. Toxicities were graded using WHO criteria (Miller et al. 1981).

\section{Treatment plan}

Gemcitabine was administered i.v. at doses of $800 \mathrm{mg} / \mathrm{m}^{2}$ on days 1 and 8 every 21 days (calculated from the first day of drug administration), as an infusion over $30 \mathrm{~min}$. In the first six patients, gemcitabine was associated with 5-fluorouracil protracted i.v. infusion at doses of $200 \mathrm{mg} / \mathrm{m}^{2} /$ daily without interruption until progression. From the seventh patient onwards, 5-fluorouracil was substituted by oral capecitabine, which was administered on a metronomic schedule at a dose of $1500 \mathrm{mg} /$ daily without interruption. Treatment was discontinued in case of unacceptable toxicity, patient's refusal or evidence of disease progression. Concomitant administration of mitotane was mandatory. Consequently, all patients received adrenal replacement therapy to prevent adrenal insufficiency.
Dose modifications were performed as follows: in case of myelosuppression (WBC count $<2.5 \times 10^{9}$ cells/ 1 and/or PLTS count $<100 \times 10^{9}$ cells/l), 5-fluorouracil and capecitabine were continued, but gemcitabine was omitted. If the blood count recovered after 1 week, gemcitabine was then administered at full dose. If the blood count had not recovered, then gemcitabine was further omitted, and the dose was subsequently reduced by $25 \%$. In the event of hand/foot syndrome, for mild to moderate palmo-plantar erythema (grade 1-2), patients continued 5-fluorouracil or capecitabine at full dose; for severe palmo-plantar erythema with blistering and desquamation, 5-fluorouracil or capecitabine was interrupted until improvement to grade $0-1$. For grade 1 or 2 diarrhea, antidiarrheal agents were prescribed; for persistent diarrhea, 5-fluorouracil or capecitabine but not gemcitabine was discontinued for 1 week. In patients with grade 2 mucositis, infusional 5-fluorouracil or capecitabine was stopped for 1 week; for grade 3/4 mucositis, all drugs were interrupted until recovery and restarted at a $25 \%$ reduction in dosage.

\section{Statistical methods}

The primary objective of the study was the evaluation of the 4-month progression-free survival (PFS) rate, which was defined as the proportion of patients without disease progression after 4 months of treatment. Secondary objectives included tumor response rate, toxicity, time to progression (TTP), which was defined as the interval between the beginning of treatment and the date of documented disease progression, and overall survival (OS), which was defined as the time from beginning of treatment until date of death or the last date of follow-up. PFS rate was evaluated both in all the patients who were registered in the study, according to the intent to treat principle, and in the patients who were considered eligible. The size of this trial was determined using the min-max two-stage phase II design by Simon (1989). Accordingly, the sample size was assessed to refuse a PFS rate of $15 \%$ (p0) after 4 months and to provide a statistical power of $80 \%$ in assessing the activity of the regimen at a PFS rate of $35 \%$ after 4 months. The upper limit of first stage rejection was 2 non-progressing patients out of 15 patients consecutively enrolled. The upper limit of second stage rejection was 7 non-progressing patients after 4 months out of 28 patients consecutively enrolled. All survival functions were computed using the Kaplan-Meier method. Statistical analysis was performed using the software STATISTICA for Windows (version 6.0) (StatSoft, Tulsa, OK, USA). 


\section{Results}

\section{Patient characteristics}

The study was activated on June 1998 and closed to patient accrual on December 2008. Twenty-nine patients entered the study and 28 of them were eligible. One patient was excluded after registration due to a diagnosis of kidney cancer after central pathology review. Table 1 shows the pretreatment characteristics of the eligible patients; they were 12 men and 16 women, aged 23-72 years. All patients had been heavily pretreated. Twenty-four patients $(85.7 \%)$ had been submitted to surgery ( 8 of them to repeated surgery), and $17(60.7 \%)$ had received mitotane either in adjuvant setting or for advanced disease. All patients had been previously submitted to one chemotherapy line with a cisplatin-containing regimen, the EDP scheme (EDP) plus mitotane in $85.8 \%$ of them. Nine patients $(32.1 \%)$ had also received second-line regimens including streptozotocin (three patients), paclitaxel (three patients), doxorubicin + lonidamine (two patients), and EDP (one patient). Hormone hypersecretion (mostly glucocorticoids with or without androgens) was demonstrated in 14 patients (50.0\%). Liver and lung were the most frequent metastatic sites, and most of the patients had at least two different sites involved.

\section{Treatment administered and toxicity}

A median of 6 months of treatment (range 2-14) was administered. All patients continued mitotane treatment (prescribed in association to previous chemotherapy) concomitantly to gemcitabine and fluoropyrimidines, at median dose of $3 \mathrm{~g} /$ day (range 1-6). Drug-related adverse events were reported in Table 2. As a whole, this chemotherapy regimen was well tolerated. Grade III and IV leukopenia was observed in six patients (21.4\%); one patient (3.5\%) experienced grade III thrombocytopenia, and one patient $(3.5 \%)$ experienced grade III mucositis. All other toxicities were mild. Dose reduction for capecitabine and gemcitabine was performed in four $(14.3 \%)$ and one patient $(3.5 \%)$ respectively, due to leukopenia and poor performance status. Dose delay or temporary discontinuation due to toxicity was required for three patients $(10.7 \%)$; gemcitabine was delayed 1 week for two patients, and capecitabine was discontinued for 1 week in one patient; leukopenia, thrombocytopenia, fever, and mucositis were the causes for these delays. Infusional 5-fluorouracil was neither reduced nor delayed because of toxicity.
Table 1 Patient characteristics

\begin{tabular}{|c|c|}
\hline$N$ & 28 \\
\hline Age (median (range)) & $45(23-72)$ \\
\hline \multicolumn{2}{|l|}{ Sex } \\
\hline Men & $12(42.9 \%)$ \\
\hline Women & $16(57.1 \%)$ \\
\hline \multicolumn{2}{|l|}{ Performance status (ECOG) } \\
\hline 0 & $15(53.6 \%)$ \\
\hline 1 & $10(35.7 \%)$ \\
\hline 2 & $3(10.7 \%)$ \\
\hline Hormone secretion & $14(50.0 \%)$ \\
\hline Glucocorticoids & $5(35.7 \%)$ \\
\hline Androgens & $3(21.5 \%)$ \\
\hline Aldosterone & $1(7.1 \%)$ \\
\hline Glucocorticoids + androgens & $3(21.5 \%)$ \\
\hline Glucocorticoids + aldosterone & $1(7.1 \%)$ \\
\hline Androgens + aldosterone & $1(7.1 \%)$ \\
\hline \multicolumn{2}{|l|}{ Disease sites } \\
\hline Lung & $15(53.7 \%)$ \\
\hline Liver & $19(67.8 \%)$ \\
\hline Adrenal & $9(32.1 \%)$ \\
\hline Lymph nodes & $10(35.7 \%)$ \\
\hline Abdomen & $2(5.2 \%)$ \\
\hline \multicolumn{2}{|l|}{ Number of sites involved } \\
\hline 1 & $8(28.6 \%)$ \\
\hline 2 & $13(46.5 \%)$ \\
\hline 3 & $6(21.4 \%)$ \\
\hline 4 & $1(3.5 \%)$ \\
\hline Previous surgery & $24(85.7 \%)$ \\
\hline 0 & $4(14.3 \%)$ \\
\hline 1 & $16(57.2 \%)$ \\
\hline 2 & $5(17.8 \%)$ \\
\hline 3 & $2(7.2 \%)$ \\
\hline 5 & $1(3.5 \%)$ \\
\hline Previous mitotane & $17(60.7 \%)$ \\
\hline Adjuvant setting & $2(11.7 \%)$ \\
\hline Advanced disease & $8(47.1 \%)$ \\
\hline Both & $7(41.2 \%)$ \\
\hline \multicolumn{2}{|l|}{ Previous chemotherapy } \\
\hline First-line chemotherapy & 28 \\
\hline $\mathrm{EDP}+$ mitotane & $24(85.8 \%)$ \\
\hline $\mathrm{DOXO}+\mathrm{P}$ & $2(7.1 \%)$ \\
\hline VP16+P & $2(7.1 \%)$ \\
\hline Second-line chemotherapy & 9 \\
\hline Streptozotocin & $3(33.3 \%)$ \\
\hline Paclitaxel & $3(33.3 \%)$ \\
\hline $\mathrm{DOXO}+\mathrm{LON}$ & $2(22.2 \%)$ \\
\hline EDP & $1(11.1 \%)$ \\
\hline
\end{tabular}

EDP, etoposide, doxorubicin, and cisplatin;

$\mathrm{DOXO}+\mathrm{P}$, doxorubicin + cisplatin; VP16 $+\mathrm{P}$, etoposide + cisplatin; DOXO + LON, doxorubicin + lonidamine.

\section{Treatment activity}

After 4 months of treatment, a clinical benefit (complete/partial response plus disease stabilization) was observed in 13 eligible patients $(46.4 \%, 95 \%$ confidence interval (CI): 27.6-65.2), while the remaining 15 patients $(53.7 \%)$ showed a disease progression 
Table 2 Toxicity (WHO criteria)

\begin{tabular}{lcccc}
\hline Event & Grade I & Grade II & Grade III & Grade IV \\
\hline $\begin{array}{l}\text { Nausea/ } \\
\quad \text { vomiting }\end{array}$ & $4(14.3 \%)$ & $1(3.5 \%)$ & - & - \\
Mucositis & $3(10.7 \%)$ & $4(14.3 \%)$ & $1(3.5 \%)$ & - \\
Hand/foot & $5(17.8 \%)$ & $2(7.1 \%)$ & - & - \\
$\quad$ syndrome & & & & \\
Diarrhea & $5(17.8 \%)$ & $2(7.1 \%)$ & - & - \\
Leukopenia & $2(7.1 \%)$ & $5(17.8 \%)$ & $5(17.8 \%)$ & $1(3.5 \%)$ \\
Anemia & $7(25.0 \%)$ & $2(7.1 \%)$ & - & - \\
Thrombo- & $2(7.1 \%)$ & $1(3.5 \%)$ & $1(3.5 \%)$ & - \\
$\quad$ cytopenia & & & & - \\
Fatigue & $7(25.0 \%)$ & $8(28.6 \%)$ & - & - \\
Myalgias & $2(7.1 \%)$ & $1(3.5 \%)$ & - & - \\
Hepatic & $2(7.1 \%)$ & $1(3.5 \%)$ & - & - \\
Neurologic & $1(3.5 \%)$ & - & - & - \\
Fever & $1(3.5 \%)$ & - & - & - \\
Conjunctivitis & $2(7.1 \%)$ & - & - & - \\
\hline
\end{tabular}

after 2-4 months (Table 3). The percent of patients obtaining a clinical benefit including all registered cases, according to an intent to treat analysis, was 44.8\% (95\% CI: 26.4-67.2). In detail, we observed 1 complete response (3.5\%), 1 partial response (3.5\%), and 11 disease stabilizations $(39.3 \%)$. The rate of disease response plus stabilization in patients receiving gemcitabine plus 5-fluorouracil (one partial response and two disease stabilizations) was similar to that obtained with gemcitabine plus capecitabine (one complete response and nine disease stabilizations). The complete response was seen in a patient with multiple liver metastases, a single vertebral metastasis, and local recurrence who attained a complete shrinkage of visceral lesions at CT scan (Fig. 1) at the second re-staging performed after 4 months, including a complete recalcification of the vertebral body lesion. The patient had been previously treated with EDP plus mitotane regimen, showing liver progression at first disease evaluation after three chemotherapy cycles. Serum mitotane levels were

Table 3 Treatment activity

\begin{tabular}{lcc}
\hline $\begin{array}{l}\text { Disease response } \\
\text { (RECIST criteria) }\end{array}$ & $\begin{array}{c}\text { Hormonal } \\
\text { response }\end{array}$ \\
\hline CR & $1(3.5 \%)$ & - \\
PR & $1(3.5 \%)$ & $3(21.4 \%)$ \\
NC & $11(39.3 \%)$ & $6(42.8 \%)$ \\
Clinical benefit & $13(46.3 \%, 95 \% \mathrm{Cl} 27.6-65.2)$ & - \\
P & $15(53.7 \%)$ & $5(35.8 \%)$ \\
\hline
\end{tabular}

$\mathrm{CR}$, complete response; $\mathrm{PR}$, partial response; NC, no change; $\mathrm{P}$, progression; $\mathrm{Cl}$, confidence interval.
$10 \mathrm{ng} / \mathrm{ml}$ at the date of first gemcitabine infusion and $16 \mathrm{mg} / \mathrm{dl}$ at the first response evaluation. At the study end, she was still disease free with a response duration of 16 months. The patient attaining a partial response was bearing a large, inoperable, abdominal mass, which initially showed a disease response to EDP plus mitotane lasting 6 months. She obtained a modest tumor shrinkage after 4 months of gemcitabine plus 5-fluorouracil that become a partial response after 6 months. Serum mitotane levels were not measured, but the amount of drug taken by the patient did not change during the study as compared to the 6 months before the enrollment. The median duration of clinical responses or disease stabilization was 6 months (range: 4-16), and individual response duration is outlined in Fig. 2. A partial hormonal response was observed in three cases (21.4\%); six patients (42.8\%) showed no change in hormone levels during treatment, while in four patients $(35.8 \%)$, hormone levels increased remarkably. All patients showing hormone response obtained a stable disease at CT scan. At the last follow-up date, 26 patients $(92.8 \%)$ showed disease progression, and $20(71.4 \%)$ patients died.

\section{Time to progression and overall survival}

Median TTP and OS in overall cases were 5.3 and 9.8 months respectively. Stratifying patients according to whether they obtained a clinical benefit or not, median survival was 15.6 and 8 months respectively. Serum mitotane levels were monitored in 19 patients, in 12 of them, the drug was above the therapeutic threshold

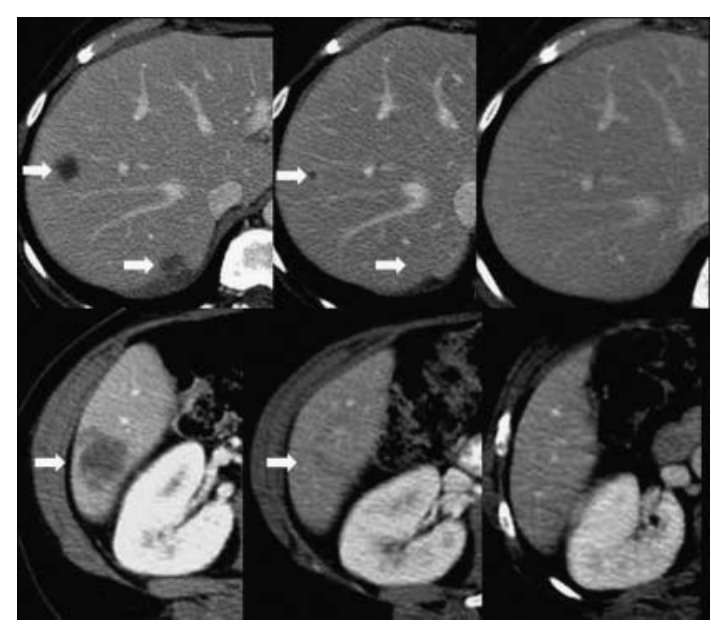

Figure 1 Complete response observed in a patient with liver metastases. As compared to baseline, CT scan after 2 months of treatment showed a partial response of liver lesions (see arrows) that completely disappeared after 4 months. 


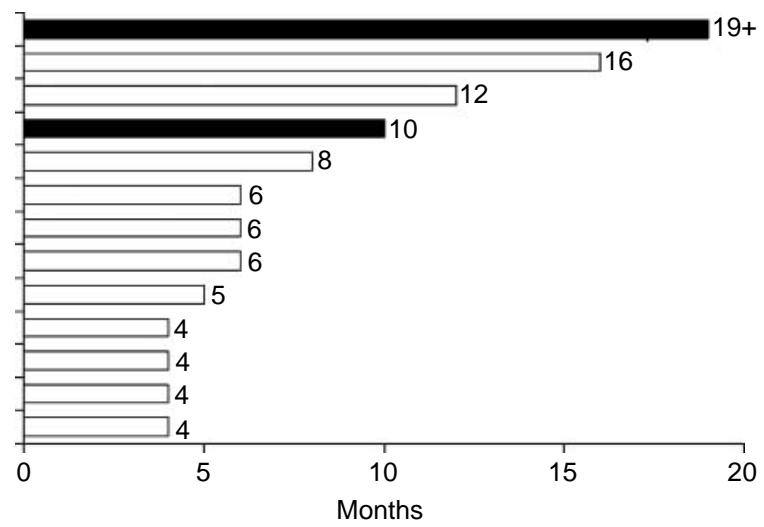

Figure 2 Duration of disease response (black bars) or stabilization (white bars) to the study treatment.

(14 ng/ml; Haak et al. 1994), while it was below the therapeutic threshold in the remaining 7 patients. Mitotane was measured as previously described (De Francia et al. 2006). As shown in Fig. 3, patients with mitotane levels above the therapeutic threshold had a longer TTP than patients with lower mitotane serum levels, just failing to attain the statistical significance $(P=0.13)$.

\section{Discussion}

ACC is poorly responsive to systemic antineoplastic treatments; therefore, the search for active salvage treatments after progression to first-/second-line chemotherapy schemes represents a difficult task. As a matter of fact, drugs or combination regimens tested previously in this clinical setting did not show any

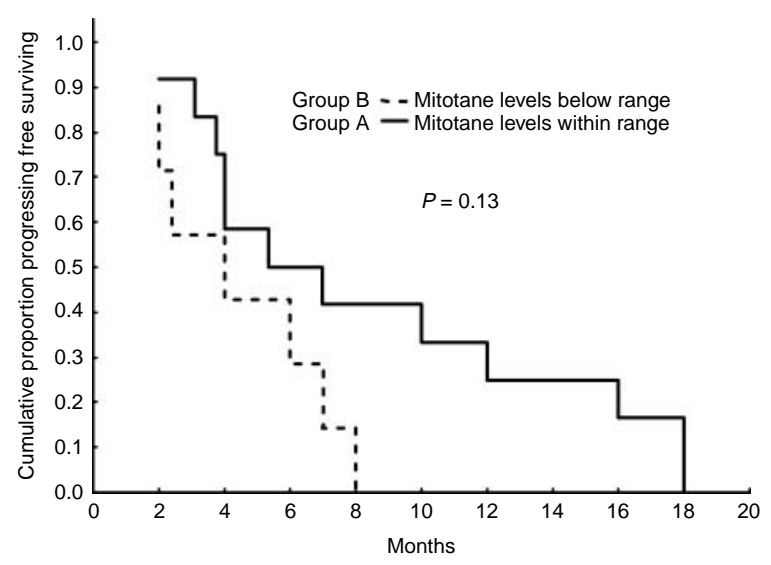

Figure 3 Time to progression curves according to mitotane concentration in 19 patients. Group A includes 12 patients whose mitotane levels were $\geq 14 \mathrm{mg} / \mathrm{l}$. Group B includes 7 patients whose mitotane levels were $<14 \mathrm{ng} / \mathrm{ml}$. relevant clinical activity (Baudin et al. 2002, Khan et al. 2004, Quinkler et al. 2008, Wortmann et al. 2009). Thus, heavily pretreated ACC patients are left with a poor life expectancy. In this scenario, not only tumor shrinkage but also disease stabilization, particularly of long duration, is clinically meaningful. This is the reason for the rate of non-progressing patients after 4 months of treatment being the primary aim of the present study and not the clinical response rate, which is a commonly employed end point in phase II chemotherapy trials. The results obtained with the association of gemcitabine and fluoropyrimidines (5-fluorouracil then substituted with oral capecitabine) are, in our opinion, encouraging. While disease response according to RECIST criteria was observed in only 2 patients (7.2\%), 13 patients $(46.4 \%)$ attained a clinical benefit in terms of disease stabilization. In the absence of a control arm, it may be difficult to discriminate whether a stable disease is attributable to the treatment efficacy or an indolent behavior of the disease. However, all patients enrolled in this trial had a documented disease progression to previous treatment regimens before being registered. The clinical benefit in our series had a prognostic significance, as demonstrated by the survival comparison between patients obtaining disease response or stabilization and patients who did not. This is an expected observation in chemotherapy trials and does not represent a surrogate of treatment efficacy (Anderson et al. 1986). In four cases $(14.3 \%)$, however, disease response or stabilization lasted between 8 and $19+$ months, and this result is noteworthy in this patient population. Interestingly, three patients with hormone-secreting ACC who had a stable disease at CT scan obtained a partial hormone response while experiencing a significant clinical improvement. One patient achieved a great result from this regimen since a long-lasting clinical complete response of the disease was obtained. Interestingly, the tumor of this patient was resistant to EDP plus mitotane; therefore, gemcitabine plus capecitabine could be potentially considered noncross resistant with cisplatin-containing regimens. This observation underlines the importance of assessing predictive markers of activity/resistance to antineoplastic agents in order to tailor to each patient the most efficacious therapy. Despite all patients included in the present trial had shown disease progression while they were on mitotane, this drug was not interrupted. Since mitotane should have been continued in the patient subset with hormone-secreting tumors, in order to obtain a homogeneous patient population, we decided to maintain this drug also in the patients without hormone-related syndromes. 
Of course, this trial does not provide any evidence that maintaining mitotane could have contributed to the positive results observed in some cases, however stratifying patients according to serum mitotane level attained, patients who had serum mitotane above the therapeutic threshold seemed to have a better TTP than those who did not. It should be noted that in the patient attaining a complete response, serum mitotane levels were just below the therapeutic range before entering the trial and attained this range at the time of response assessment, so that we could not exclude a possible contribution of mitotane to the response obtained. These data suggest to explore the efficacy of continuing mitotane in addition to new chemotherapeutic drugs in patients experiencing progression during an (Weiss 1984) initial mitotane-based regimen. Gemcitabine and fluoropyrimidine combination regimen was well tolerated; leukopenia and mucositis were the most severe toxicity observed, but only rarely they led to dose reduction and temporarily treatment withdrawal. The non-overlapping toxicity profile of gemcitabine and 5-fluorouracil or capecitabine could account for the good tolerability of the combination. The substitution of infusional 5-fluorouracil with oral capecitabine, avoiding the need of a central venous access, improved overall tolerability. The rarity of this tumor hampers the implementation of clinical trials. As a matter of fact, the patient accrual in this trial was very long (10 years), and this represents a limitation. Recently, it has been shown that either gemcitabine administered in association to erlotinib (Quinkler et al. 2008) or capecitabine administered in association to bevacizumab (Wortmann et al. 2009) led to poor results in heavily treated patients. These data seem in contrast with the results of the present study. We do not have any clear explanation of these differences but only suggestions. Epidermal growth factor receptor inhibitors and vascular endothelial growth factor inhibitors may be not efficacious in the management of ACC patients, despite the positive results obtained in other malignancies. Gemcitabine and capecitabine may be not very active when used alone, but the association of drugs can increase the efficacy. Gemcitabine and capecitabine (or 5-fluorouracil), in fact, inhibit different targets and show synergistic antitumor activity when combined in human xenograft models (Sawada et al. 2002).

In conclusion, gemcitabine plus metronomic fluoropyrimidines is a well-tolerated regimen for heavily pretreated ACC patients and is potentially able to delay the disease progression in a substantial proportion of patients.

\section{Declaration of interest}

The authors declare that there is no conflict of interest that could be perceived as prejudicing the impartiality of the research reported.

\section{Funding}

This work was supported by a grant from the Ricerca Sanitaria Finalizzata, Regione Piemonte March 2008.

\section{References}

Abiven G, Coste J, Groussin L, Anract P, Tissier F, Legmann P, Dousset B, Bertagna X \& Bertherat J 2006 Clinical and biological features in the prognosis of adrenocortical cancer: poor outcome of cortisolsecreting tumors in a series of 202 consecutive patients. Journal of Clinical Endocrinology and Metabolism 91 2650-2655.

Abraham J, Bakke S, Rutt A, Meadows B, Merino M, Alexander R, Schrump D, Bartlett D, Choyke P, Robey R et al. 2002 A phase II trial of combination chemotherapy and surgical resection for the treatment of metastatic adrenocortical carcinoma: continuous infusion doxorubicin, vincristine, and etoposide with daily mitotane as a P-glycoprotein antagonist. Cancer 94 2333-2343.

Allolio B \& Fassnacht M 2006 Clinical review: adrenocortical carcinoma: clinical update. Journal of Clinical Endocrinology and Metabolism 91 2027-2037.

Anderson JR, Ginsberg S \& Gottlieb AJ 1986 Chemotherapy of diffuse large-cell lymphoma - rapidly responding patients have more durable remissions. Journal of Clinical Oncology 4 1420-1421.

Baudin E, Docao C, Gicquel C, Vassal G, Bachelot A, Penfornis A \& Schlumberger M 2002 Use of a topoisomerase I inhibitor (irinotecan, CPT-11) in metastatic adrenocortical carcinoma. Annals of Oncology 13 1806-1809.

Berruti A, Sperone P, Bottini A, Gorzegno G, Lorusso V, Brunelli A, Botta M, Tampellini M, Donadio M, Mancarella S et al. 2000 Phase II study of vinorelbine with protracted fluorouracil infusion as a second- or third-line approach for advanced breast cancer patients previously treated with anthracyclines. Journal of Clinical Oncology 18 3370-3377.

Berruti A, Terzolo M, Sperone P, Pia A, Casa SD, Gross DJ, Carnaghi C, Casali P, Porpiglia F, Mantero F et al. 2005 Etoposide, doxorubicin and cisplatin plus mitotane in the treatment of advanced adrenocortical carcinoma: a large prospective phase II trial. Endocrine-Related Cancer 12 657-666.

Bonacci R, Gigliotti A, Baudin E, Wion-Barbot N, Emy P, Bonnay M, Cailleux AF, Nakib I \& Schlumberger M 1998 Cytotoxic therapy with etoposide and cisplatin in advanced adrenocortical carcinoma. British Journal of Cancer 78 546-549. 
Bottini A, Generali D, Brizzi MP, Fox SB, Bersiga A, Bonardi S, Allevi G, Aguggini S, Bodini G, Milani M et al. 2006 Randomized phase II trial of letrozole and letrozole plus low-dose metronomic oral cyclophosphamide as primary systemic treatment in elderly breast cancer patients. Journal of Clinical Oncology 24 3623-3628.

Bukowski RM, Wolfe M, Levine HS, Crawford DE, Stephens RL, Gaynor E \& Harker WG 1993 Phase II trial of mitotane and cisplatin in patients with adrenal carcinoma: a Southwest Oncology Group Study. Journal of Clinical Oncology 11 161-165.

Crucitti F, Bellantone R, Ferrante A, Boscherini M \& Crucitti P 1996 The Italian Registry for Adrenal Cortical Carcinoma: analysis of a multiinstitutional series of 129 patients. The ACC Italian Registry Study Group. Surgery 119 161-170.

Decker RA, Elson P, Hogan TF, Citrin DL, Westring DW, Banerjee TK, Gilchrist KW \& Horton J 1991 Eastern Cooperative Oncology Group Study 1879: mitotane and adriamycin in patients with advanced adrenocortical carcinoma. Surgery 110 1006-1013.

De Francia S, Pirro E, Zappia F, De Martino F, Sprio AE, Daffara F, Terzolo M, Berruti A, Di Carlo F \& Ghezzo F 2006 A new simple HPLC method for measuring mitotane and its two principal metabolites. Tests in animals and mitotane-treated patients. Journal of Chromatography. B. Analytical Technologies in the Biomedical and Life Sciences 837 69-75.

Fassnacht M, Johanssen S, Quinkler M, Bucsky P, Willenberg HS, Beuschlein F, Terzolo M, Mueller HH, Hahner S \& Allolio B 2009 Limited prognostic value of the 2004 International Union Against Cancer staging classification for adrenocortical carcinoma: proposal for a Revised TNM Classification. Cancer 115 243-250.

Haak HR, Hermans J, van de Velde CJ, Lentjes EG, Goslings BM, Fleuren GJ \& Krans HM 1994 Optimal treatment of adrenocortical carcinoma with mitotane: results in a consecutive series of 96 patients. British Journal of Cancer 69 947-951.

Hahner S \& Fassnacht M 2005 Mitotane for adrenocortical carcinoma treatment. Current Opinion in Investigational Drugs 6 386-394.

Herrmann R, Bodoky G, Ruhstaller T, Glimelius B, Bajetta E, Schuller J, Saletti P, Bauer J, Figer A, Pestalozzi B et al. 2007 Gemcitabine plus capecitabine compared with gemcitabine alone in advanced pancreatic cancer: a randomized, multicenter, phase III trial of the Swiss Group for Clinical Cancer Research and the Central European Cooperative Oncology Group. Journal of Clinical Oncology 25 2212-2217.

Icard P, Goudet P, Charpenay C, Andreassian B, Carnaille B, Chapuis Y, Cougard P, Henry JF \& Proye C 2001 Adrenocortical carcinomas: surgical trends and results of a 253-patient series from the French Association of Endocrine Surgeons Study Group. World Journal of Surgery 25 891-897.

Kerbel RS \& Kamen BA 2004 The anti-angiogenic basis of metronomic chemotherapy. Nature Reviews. Cancer 4 423-436.

Khan TS, Imam H, Juhlin C, Skogseid B, Grondal S, Tibblin S, Wilander E, Oberg K \& Eriksson B 2000 Streptozocin and $o, p^{\prime}$ DDD in the treatment of adrenocortical cancer patients: long-term survival in its adjuvant use. Annals of Oncology 11 1281-1287.

Khan TS, Sundin A, Juhlin C, Wilander E, Oberg K \& Eriksson B 2004 Vincristine, cisplatin, teniposide, and cyclophosphamide combination in the treatment of recurrent or metastatic adrenocortical cancer. Medical Oncology 21 167-177.

Miller AB, Hoogstraten B, Staquet M \& Winkler A 1981 Reporting results of cancer treatment. Cancer 47 207-214.

Quinkler M, Hahner S, Wortmann S, Johanssen S, Adam P, Ritter C, Strasburger C, Allolio B \& Fassnacht M 2008 Treatment of advanced adrenocortical carcinoma with erlotinib plus gemcitabine. Journal of Clinical Endocrinology and Metabolism 93 2057-2062.

Sawada N, Fujimoto-Ouchi K \& Ishikawa T 2002 Antitumor activity with capecitabine plus vinorelbine, and capecitabine plus gemcitabine in human tumor xenograft models. Proceedings of the American Association for Cancer Research 43 abstract 5388.

Schlumberger M, Brugieres L, Gicquel C, Travagli JP, Droz JP \& Parmentier C 1991 5-Fluorouracil, doxorubicin, and cisplatin as treatment for adrenal cortical carcinoma. Cancer 67 2997-3000.

Schteingart DE, Doherty GM, Gauger PG, Giordano TJ, Hammer GD, Korobkin M \& Worden FP 2005 Management of patients with adrenal cancer: recommendations of an international consensus conference. Endocrine-Related Cancer 12 667-680.

Simon R 1989 Optimal two-stage designs for phase II clinical trials. Controlled Clinical Trials 10 1-10.

van Slooten H \& van Oosterom AT 1983 CAP (cyclophosphamide, doxorubicin, and cisplatin) regimen in adrenal cortical carcinoma. Cancer Treatment Reports 67 377-379.

Tannir NM, Thall PF, Ng CS, Wang X, Wooten L, SiefkerRadtke A, Mathew P, Pagliaro L, Wood C \& Jonasch E 2008 A phase II trial of gemcitabine plus capecitabine for metastatic renal cell cancer previously treated with immunotherapy and targeted agents. Journal of Urology 180 867-872.

Therasse P, Arbuck SG, Eisenhauer EA, Wanders J, Kaplan RS, Rubinstein L, Verweij J, Van Glabbeke M, van Oosterom AT, Christian MC et al. $2000 \mathrm{New}$ guidelines to evaluate the response to treatment in solid tumors, European Organization for Research and Treatment of Cancer, National Cancer Institute of the United States, National Cancer Institute of Canada. Journal of the National Cancer Institute 92 205-216. 
Weiss LM 1984 Comparative histologic study of 43 metastasizing and nonmetastasizing adrenocortical tumors. American Journal of Surgical Pathology 8 163-169.

Williamson SK, Lew D, Miller GJ, Balcerzak SP, Baker LH \& Crawford ED 2000 Phase II evaluation of cisplatin and etoposide followed by mitotane at disease progression in patients with locally advanced or metastatic adrenocortical carcinoma: a Southwest Oncology Group Study. Cancer 88 1159-1165.

Wortmann S, Quinkler M, Ritter C, Kroiss M, Johanssen S, Hahner S, Allolio B \& Fassnacht M 2009 Bevacizumab plus capecitabine as a salvage therapy in advanced adrenocortical carcinoma. European Journal of Endocrinology 162 349-356. 